\title{
McGill Journal of Education
}

Revue des sciences de l'éducation de McGill

\section{DE LA FABRICATION DE L'EXCLUSION SCOLAIRE EN MILIEU DÉFAVORISÉ ET MULTIETHNIQUE THE MAKING OF SCHOOL EXCLUSION IN A POOR AND MULTIETHNIC SETTING}

\section{Marjorie Vidal}

Volume 54, Number 2, Spring 2019

Understanding Exclusion to Better Work Towards Inclusion?

Comprendre l'exclusion pour mieux tendre vers l'inclusion?

URI: https://id.erudit.org/iderudit/1065655ar

DOI: https://doi.org/10.7202/1065655ar

See table of contents

Publisher(s)

Faculty of Education, McGill University

ISSN

1916-0666 (digital)

Explore this journal

Cite this article

Vidal, M. (2019). DE LA FABRICATION DE L'EXCLUSION SCOLAIRE EN MILIEU DÉFAVORISÉ ET MULTIETHNIQUE. McGill Journal of Education / Revue des sciences de l'éducation de McGill, 54(2), 206-225.

https://doi.org/10.7202/1065655ar

\section{Article abstract}

This article aims to understand the making of school exclusion by studying organizational social capital. It is based on an ethnographic case study that took place in a secondary school in a poor and multiethnic area in Montreal. The use of " school form " revealed how some practices that promote academic excellence and focus on school culture can create obstacles to inclusion, by reproducing a norm that contributes to exclude students in vulnerable situations.
(C) Faculty of Education, McGill University, 2019
This document is protected by copyright law. Use of the services of Érudit (including reproduction) is subject to its terms and conditions, which can be viewed online.

https://apropos.erudit.org/en/users/policy-on-use/ 


\section{DE LA FABRICATION DE L'EXCLUSION SCOLAIRE EN MILIEU DÉFAVORISÉ ET MULTIETHNIQUE}

MARJORIE VIDAL Université du Québec à Montréal

RÉSUMÉ. Cet article a pour objectif de comprendre la fabrication de l'exclusion scolaire, à travers l'étude du capital social organisationnel. Il repose sur une étude de cas ethnographique qui s'est déroulée dans une école secondaire en milieu défavorisé et multiethnique de Montréal. Le recours à la " forme scolaire " a permis de révéler comment certaines pratiques qui relèvent de la promotion d'un parcours d'excellence, ou encore de la centration sur la culture scolaire, constituent des obstacles à la véritable inclusion, en perpétuant une norme qui contribue à exclure les élèves en situation de vulnérabilité.

\section{THE MAKING OF SCHOOL EXCLUSION IN A POOR AND MULTIETHNIC SETTING}

ABSTRACT. This article aims to understand the making of school exclusion by studying organizational social capital. It is based on an ethnographic case study that took place in a secondary school in a poor and multiethnic area in Montreal. The use of "school form » revealed how some practices that promote academic excellence and focus on school culture can create obstacles to inclusion, by reproducing a norm that contributes to exclude students in vulnerable situations.

Intégration, non-intégration, inclusion, exclusion. Ces termes, dans l'idée d'opposer des individus par rapport à la représentation d'une organisation sociale ou d'une institution, ont fait couler beaucoup d'encre dans les cercles sociologique et praxéologique, notamment parce qu'ils questionnent le lien social, la cohésion sociale, sur la base d'une distinction idéalisée, voire fantasmée, et souvent porteuse de préjugés, entre un groupe majoritaire et un groupe minoritaire. La théorie fonctionnaliste a, par le passé, tenté d'expliquer cette distinction, appréhendant les étrangers au groupe social majoritaire - ceux que Becker nommait déjà en 1963 les " outsiders " - comme des déviants, c'est-à-dire des individus dysfonctionnels non conformes à la norme. Selon cette perspective, l'inclusion reposerait sur la capacité des personnes (par leur motivation ou leurs compétences) à se conformer à ce qui est attendu d'eux; les déviants - les exclus - étant ceux qui ne peuvent ou ne veulent pas se 
soumettre à la norme. L'école, à travers sa fonction de sélection des élèves (Parsons, 1959; Tumin, 1967) aurait historiquement encouragé et reconduit une forme de validation de cette norme (Tondreau et Robert, 2011).

Depuis, Becker (1963) a ouvert une brèche avec sa théorie de l'étiquetage. Dans son ouvrage Outsiders, il critique ouvertement l'approche fonctionnaliste en rejetant l'idée selon laquelle la déviance se réduirait aux simples caractéristiques de la personne considérée comme telle. Pour lui, la déviance est beaucoup plus complexe. C'est une construction sociale qui se crée en interaction entre les déviants, d'une part, et ceux qui les caractérisent comme tels, de l'autre. Par conséquent, le jugement de ceux qui attribuent l'étiquette de déviant et leur mobilisation pour ne pas inclure les individus qui portent cette étiquette (ceux que Becker appelle des "entrepreneurs de la morale ») font partie intégrante du phénomène d'exclusion. Ce processus de catégorisation se retrouve également dans les interactions scolaires (Magnan, Pilote, Vidal et Collins, 2016). À titre d'exemple, Perrenoud (1990) avance qu'on pardonnera davantage à l'élève poli, qui se comporte selon les attentes, plutôt qu'à celui qui jure, sent mauvais et s'en prend aux plantes du maître, quand bien même les deux élèves seraient à compétences égales!

Le changement apporté par Becker a plusieurs implications épistémologiques au regard des personnes vulnérables. ${ }^{1}$ Une telle perspective oblige à adopter un point de vue interactif et à considérer dans l'équation ceux qui sont à l'origine même de la construction de l'exclusion. ${ }^{2}$ Ce changement invite désormais les acteurs du système, dont l'école fait partie, à questionner leurs représentations, à moduler leurs exigences et à s'adapter face à la diversité des publics et des situations, puisque la personne exclue n'est plus vue comme la seule responsable de son exclusion. Il s'agit pour l'école d'offrir des services appropriés aux besoins des personnes issues des groupes minoritaires, selon les principes d'équité (Ministère de l'Éducation, des Loisirs et du Sport [MELS], 2007). En éducation, l'adaptation à la diversité des publics devient une exigence et s'inscrit désormais dans les cadres règlementaires dès la fin des années 1990. Ils doivent permettre aux élèves en situation de handicap (EHDAA) ou issus de l'immigration d'être scolarisés dans le contexte le plus " normal " possible, en fonction de leurs besoins (MELS, 2007; Ministère de l'éducation du Québec [MEQ], 1999, 1998).

Autre implication, les processus d'exclusion et d'inclusion se construisent avant tout sur le terrain, c'est-à-dire au travers de situations, d'interactions et de pratiques entre les différents acteurs scolaires (direction, enseignants, parents, élèves, etc.; Tondreau et Robert, 2011). En ce sens, plusieurs pratiques scolaires peuvent être considérées comme inclusives car elles rejoignent tous les élèves, et notamment les plus vulnérables. Ces pratiques offrent une représentation "dénormalisée " de la réalité (AuCoin et Vienneau, 2015) puisqu'elles permettent de prendre en compte les besoins et réalités spécifiques des élèves sans que ceux-ci doivent les modifier (AuCoin et Vienneau, 2015; 
Conseil supérieur de l'éducation [CSE], 2017). Elles concernent aussi bien la pédagogie (Prud'homme, 2015), l'intervention en orthopédagogie (Lemire, 2010) et en orthophonie (Borri-Anadon, 2015), l'intervention psychosociale (Institut national d'excellence en santé et en services sociaux [INESSS], 2018) ou encore la gestion et l'administration scolaire (Archambault, Garon et Harnois, 2011; Deniger, 2012).

Par effet miroir, il existe également des pratiques exclusives. Elles perpétuent une certaine norme institutionnelle qui ne leur permet pas de rejoindre les élèves les plus vulnérables, participant ainsi de leur marginalisation. C'est la présentation de certaines de ces pratiques que cet article propose. En effet, mieux comprendre les processus mis en œuvre dans l'exclusion devrait favoriser la réflexion concernant les obstacles qui se dressent sur le chemin de la véritable inclusion, celle qui tient compte des réalités et des besoins de tous les élèves. Le présent article s'appuie sur les résultats d'une recherche qui avait pour objectif de comprendre la production de capital social dans une école secondaire en milieu défavorisé et multiethnique : il s'agit des relations auxquelles les individus ont accès dans leurs réseaux (Lin, 1995), et qui leur permettent d'accéder à des ressources économiques, culturelles, symboliques et également à d'autres ressources sociales. Le recours au concept de forme scolaire a permis de s'intéresser au capital social sur le plan organisationnel, c'est-à-dire d'interroger la capacité de l'école secondaire à mettre en place les conditions pour rejoindre les élèves en situation de vulnérabilité (Vidal, 2017). Aussi, la première partie de cet article fait état du contexte de la recherche. La deuxième partie en présente les cadres théorique et méthodologique. La troisième partie révèle enfin les principaux résultats concernant les pratiques scolaires qui mènent à une forme d'exclusion des élèves vulnérables.

\section{L'EXCLUSION COMME ABSENCE DE PRODUCTION DE CAPITAL SOCIAL}

Le milieu d'apprentissage étant un milieu fondamentalement social (Duru-Bellat, 2002), les relations qui s'y développent sont d'autant plus significatives. Les facteurs de nature sociale font en effet partie des facteurs les plus importants en matière éducative (Perrenoud, 1994). Le capital social présente un intérêt certain, puisqu'il permet d'appréhender les relations des individus comme des ressources sociales, c'est-à-dire comme des biens qui peuvent être mobilisés à des fins instrumentales (Lin, 1995). L'investissement dans ces ressources sociales entraînerait des bénéfices accrus à plusieurs niveaux (Portes, 1998; Zhang, DeBlois, Deniger et Kamanzi, 2008). À l'échelle de l'organisation scolaire - c'està-dire des relations entre les directeurs, les administrateurs, les enseignants, les élèves et les parents - le capital social renforcerait la confiance et la cohésion de groupe; faciliterait la transmission de l'information et la communication; contribuerait à une vision commune et augmenterait les effets positifs dans les classes, la compétence des enseignants et l'appropriation du changement (Leana et Pil, 2014; Pil et Leana, 2009; Quintero, 2014; Spillane, 2015). 
Le capital social a également des effets bénéfiques sur la réussite des élèves. La littérature scientifique regorge d'études qui se sont penchées sur les liens entre capital social et réussite scolaire (Portes, 1998), que celle-ci prenne la forme de la persévérance scolaire (diplôme et passage aux cycles supérieurs), de la performance scolaire (résultats scolaires) ou encore des conduites proacadémiques comme la motivation, les aspirations scolaires ou la participation aux activités scolaires et parascolaires (Dika et Singh, 2002; Kamanzi, Zhang, Deblois et Deniger, 2007; White et Glick, 2000). Il appert que, pour les élèves qui présentent des conditions initiales défavorables, le lien entre capital social et réussite est particulièrement important. Ces élèves (et leurs parents) posséderaient en effet un capital social différent de celui préconisé dans le milieu scolaire, et auraient ainsi plus de difficulté à en comprendre les normes, comportements et attitudes attendus (Lareau et Dorvat, 1999). Ce serait notamment le cas des élèves provenant de milieux défavorisés ou encore issus des minorités ethniques (Kamanzi et coll., 2007; Stanton-Salazar, 1997; Yan, 1999).

Il semble que, pour ces élèves, le capital social de l'école puisse jouer un rôle compensatoire en leur donnant accès à des ressources économiques, culturelles, symboliques et sociales auxquelles ces élèves n'auraient pas accès autrement, contrairement aux familles de milieu socioéconomique favorisé. Il s'agit par exemple des objets matériels (ordinateur, livres, etc.), des activités culturelles (cinéma, théâtre, jeux, etc.) ou encore des valeurs symboliques (des aspirations scolaires et professionnelles, des satisfactions; Kamanzi et coll., 2007). Zhang et coll. (2008) et Yan (1999) affirment à cet égard que les chances de réussite scolaire des élèves issus de milieux défavorisés augmentent proportionnellement avec le capital social de l'école. Le capital social de l'école jouerait un rôle d'autant plus important lorsque ces élèves qui présentent des conditions initiales défavorables rencontrent des difficultés dans leur parcours scolaire, notamment au cours des périodes critiques comme des évaluations ou encore des transitions scolaires (Bevort et Lallement, 2006; Lareau et Dorvat, 1999; Stanton-Salazar, 1997).

L'intérêt de cette approche est de voir le capital social de l'école comme un levier pour l'intervention auprès des élèves en situation de vulnérabilité. Dans une perspective d'intervention scolaire, il s'agit plus précisément de voir les différentes ressources sociales de l'école comme un moyen d'intervenir efficacement auprès des élèves qui en ont le plus besoin. Il peut s'agir par exemple de l'enseignant, de l'orthopédagogue, de l'intervenant psychosocial, du technicien en éducation spécialisée, ou de tout acteur dans le cadre scolaire qui pourrait jouer un rôle auprès de ces élèves, comme les pairs. Toutefois, pour pouvoir appréhender justement le phénomène de capital social dans l'école, il est nécessaire de distinguer le capital social accessible, c'est-à-dire l'ensemble des ressources sociales mises à disposition des élèves, du capital social produit, c'est-à-dire les ressources effectivement mobilisées par les élèves. La partie 
suivante montre que cette distinction est en effet nécessaire pour pouvoir interroger la capacité de l'école à mettre en place les conditions favorables à cette production de capital social.

\section{LE CAPITAL SOCIAL ORGANISATIONNEL : LA CAPACITÉ DE L'ÉCOLE À REJOINDRE LES ÉLĖVES VULNÉRABLES}

La recherche sur laquelle s'appuie cet article repose sur une étude de cas ethnographique menée dans une école secondaire de Montréal. L'objectif de la recherche était d'étudier de manière exhaustive la production de capital social, c'est-à-dire la mobilisation effective des ressources sociales par les élèves vulnérables, dans le contexte d'un milieu scolaire urbain, défavorisé et multiethnique. Le recours au concept de forme scolaire a permis de s'intéresser à l'organisation du capital social du point de vue de l'institution, et ainsi d'interroger la capacité de l'école à mettre en place les conditions pour aider les élèves en situation de vulnérabilité à mobiliser les ressources auxquelles ils ont accès. C'est de ce dernier aspect que nous rendons compte dans cet article. Cette partie présente les choix méthodologiques et théoriques qui ont guidé la recherche.

\section{La collecte de données}

L'école investiguée - nommée Pierre-Mathieu (nom fictif) - est une grande école secondaire de Montréal qui accueille entre 900 et 1000 élèves. ${ }^{3}$ Il s'agit d'une école pluriethnique puisque plus de $75 \%$ des élèves sont nés à l'étranger (immigration de première génération) ou sont nés au Québec de deux parents nés ailleurs (immigration de deuxième génération). À cela s'ajoute qu'à peine le quart des élèves ont le français comme langue maternelle et $22 \%$ ont le créole comme langue maternelle. Selon les indices socioculturels de défavorisation du ministère de l'Éducation et de l'Enseignement supérieur (MEES, 2017a), l'école se situe dans un quartier très défavorisé. ${ }^{4}$ Le choix du milieu investigué est également tributaire de ses caractéristiques qui en font une école dite difficile : mal classée dans le Palmarès des écoles secondaires (Institut Fraser, 2016), elle présente un fort taux de décrochage scolaire et accueille une population d'élèves qui pourraient être qualifiés comme présentant des difficultés scolaires, avec plus de $45 \%$ des élèves en retard scolaire, $40 \%$ en échec et environ $50 \%$ des élèves catégorisés HDAA (Institut Fraser, 2016).

L'étude de cas ethnographique repose sur une variété de méthodes de collecte de données (Merriam, 1998). Elles ont constitué autant d'étapes vers une appréhension plus fine de la production de capital social dans l'école. La principale méthode a consisté en des observations, qui se sont déroulées sur 40 mois (200 jours d'observation). Les différents outils de collecte de données ont été articulés autour des observations, qui constituent le fil directeur de la recherche. D'une part, les observations des événements significatifs concernant les acteurs scolaires, ${ }^{5}$ l'étude des documents institutionnels ${ }^{6}$ et les neuf 
entretiens spontanés avec les personnes-clés dans l'école ${ }^{7}(n=8$ participants) ont permis de renseigner le capital social accessible, c'est-à-dire tout ce qui était en lien avec les ressources mises à disposition des élèves. Concernant plus précisément les entretiens spontanés, il s'agissait d'entretiens sur le vif auprès de personnes qui tenaient une position centrale au sein de l'école. Ces entretiens ont ainsi aidé à approfondir la compréhension d'une situation, à fournir des renseignements manquants qui devaient être vérifiés par la suite, ou à faire remarquer si une situation était routinière ou plutôt extraordinaire, dans le sens où elle venait justement briser la routine installée.

D'autre part, les observations d'événements significatifs avec les élèves, ${ }^{8}$ les questionnaires sur leurs réseaux sociaux soumis aux participants $^{9}(n=16)$ et deux entretiens de groupe avec les élèves ( $n=9$ participants), qui ont duré $1 \mathrm{~h} 10$ et $1 \mathrm{~h} 20$, ont permis de renseigner le capital social produit, c'est-à-dire leur mobilisation et leurs représentations ${ }^{10}$ de ces ressources. Les 19 élèves qui ont participé à la recherche ont été sélectionnés sur la base de la variété de leurs profils. Le Tableau 1 reproduit les méthodes de collecte de données selon les élèves participants à la recherche.

\section{L'analyse des données}

L'analyse thématique (Paillé et Mucchielli, 2012) a été guidée par l'étude des obstacles identifiés entre capital social accessible et capital social mobilisé. Il pouvait s'agir de représentations négatives envers certaines fonctions (par exemple, le conseiller pédagogique), d'une méconnaissance et d'un rejet de certaines ressources (plusieurs enseignants de français notamment), d'un manque d'articulation de certaines ressources avec le projet des élèves, d'un accent mis sur l'évaluation ou encore du fait que les élèves estimaient que leur marge de manœuvre était trop faible dans certaines situations. Ces différentes situations ont été identifiées comme des freins à la mobilisation des ressources quand les élèves rencontraient des difficultés dans leurs parcours. Le recours au concept de forme scolaire a permis d'opérer un changement de point de vue sur ces différentes situations et de passer de nouveau le matériel au crible : il s'agissait cette fois-ci d'interroger l'école et sa capacité à mettre en place les conditions nécessaires pour favoriser la production de capital social, c'est-à-dire la mobilisation de ces ressources, et donc de rejoindre les élèves en situation de vulnérabilité.

Vincent (1980) est à l'origine du concept de forme scolaire qu'il appréhende comme un mode de socialisation spécifique à l'organisation scolaire (à travers notamment l'imposition de règles, l'organisation d'un temps de travail spécifique, la répétition d'exercices). L'école y est envisagée comme un acteur important dans le maintien et la reproduction du pouvoir, puisqu'elle incite les acteurs scolaires, et surtout les élèves, à s'approprier les normes sociales dominantes dont elle fait elle-même la promotion. La forme scolaire participe ainsi d'une domination rationnelle légale; elle traduit l'apprentissage des relations de pouvoir 
TABLEAU I. Le profil des élèves et les différentes méthodes de collecte de données

\begin{tabular}{|c|c|c|c|c|c|c|}
\hline Nom & Âge & Classe & $\begin{array}{c}\text { Vague } \\
\text { migratoire }\end{array}$ & $\begin{array}{c}\text { Pays } \\
\text { d'origine }\end{array}$ & EHDAA & Modalités méthodologiques \\
\hline Eddy & 17 & Adaptation & $2^{\mathrm{e}}$ génération & Haïti & $?^{\mathrm{a}}$ & Observations et entretiens \\
\hline Yacine & 17 & Adaptation & $2^{\mathrm{e}}$ génération & Algérie & $\mathrm{TC}$ & Observations \\
\hline Antoine & 17 & $\begin{array}{l}\text { Adaptation } \\
\text { et renvoi }\end{array}$ & Canada (QC) & & $\mathrm{TC}$ & Observations \\
\hline Parfait & ? & Adaptation & $2^{\mathrm{e}}$ génération & Haïti & $\mathrm{TC}$ & Observations \\
\hline Brandon & 13 & Adaptation & $2^{e}$ génération & Salvador & TA & $\begin{array}{l}\text { Observations, questionnaires } \\
\text { et entretiens }\end{array}$ \\
\hline Dagobert & $?$ & Adaptation & $2^{\mathrm{e}}$ génération & Haïti & ? & $\begin{array}{l}\text { Observations et question- } \\
\text { naires }\end{array}$ \\
\hline Dario & 16 & $\begin{array}{l}\text { Régulier : } \\
\text { secondaire } 4\end{array}$ & $2^{\mathrm{e}}$ génération & $\begin{array}{l}\text { Salvador et } \\
\text { Guatemala }\end{array}$ & - & $\begin{array}{l}\text { Observations, questionnaires } \\
\text { et entretiens }\end{array}$ \\
\hline Danny & 12 & $\begin{array}{l}\text { Régulier : } \\
\text { secondaire } 1\end{array}$ & Canada (QC) & & TA & $\begin{array}{l}\text { Observations, questionnaires } \\
\text { et entretiens }\end{array}$ \\
\hline Eduardo & 15 & $\begin{array}{l}\text { Régulier : } \\
\text { secondaire } 4\end{array}$ & $2^{\mathrm{e}}$ génération & Costa Rica & - & $\begin{array}{l}\text { Observations, questionnaires } \\
\text { et entretiens }\end{array}$ \\
\hline Gaens & 15 & $\begin{array}{l}\text { Régulier : } \\
\text { secondaire } 4\end{array}$ & $1^{\mathrm{re}}$ génération & Haïti & - & Observations \\
\hline Michael & 12 & $\begin{array}{l}\text { Régulier : } \\
\text { secondaire } 1\end{array}$ & $2^{\mathrm{e}}$ génération & Haiti & - & $\begin{array}{l}\text { Observations, questionnaires } \\
\text { et entretiens }\end{array}$ \\
\hline $\begin{array}{l}\text { Mah- } \\
\text { moud }\end{array}$ & 15 & $\begin{array}{l}\text { Régulier : } \\
\text { secondaire } 4\end{array}$ & $2^{\mathrm{e}}$ génération & Haïti & - & $\begin{array}{l}\text { Observations, questionnaires } \\
\text { et entretiens }\end{array}$ \\
\hline Mabrouk & 16 & $\begin{array}{l}\text { Régulier et } \\
\text { renvoi }\end{array}$ & $2^{\mathrm{e}}$ génération & Maroc & - & Observations \\
\hline Pascal & 16 & $\begin{array}{l}\text { Régulier : } \\
\text { secondaire } 4\end{array}$ & $2^{\mathrm{e}}$ génération & Haïti & - & Observations \\
\hline Roberto & 17 & Adaptation & Canada (QC) & & TA & $\begin{array}{l}\text { Observations, questionnaires } \\
\text { et entretiens }\end{array}$ \\
\hline $\begin{array}{l}\text { Saint- } \\
\text { Martin }\end{array}$ & 13 & Adaptation & $2^{\mathrm{e}}$ génération & Haïti & TA & $\begin{array}{l}\text { Observations, questionnaires } \\
\text { et entretiens }\end{array}$ \\
\hline Toufik & 15 & $\begin{array}{l}\text { Régulier : } \\
\text { secondaire } 3\end{array}$ & $2^{\mathrm{e}}$ génération & Maroc & - & $\begin{array}{l}\text { Observations, questionnaires } \\
\text { et entretiens }\end{array}$ \\
\hline Yael & 14 & Adaptation & Canada (QC) & & TA & $\begin{array}{l}\text { Observations, questionnaires } \\
\text { et entretiens }\end{array}$ \\
\hline Yassim & 15 & Adaptation & Canada (QC) & & TA & Observations \\
\hline
\end{tabular}

NOTES. $T C=$ troubles du comportement; $T A=$ troubles d'apprentissage.

a'Linformation n'est pas donnée par l'élève ou semble inexacte (selon la triangulation des données). 
à travers une socialisation légitimée, parce que considérée comme représentant la réalité (Vincent, 2008). Dans le cadre de notre recherche, le concept de forme scolaire a permis de réinterroger la production du capital social sous l'angle particulier de l'organisation scolaire. Il est ainsi apparu que plusieurs des obstacles soulevés pouvaient se rattacher à des pratiques potentiellement exclusives, en ce sens qu'elles reproduisaient une certaine norme scolaire génératrice de rapports de pouvoir qui pouvait contribuer à exclure certains élèves en situation de vulnérabilité. Ce sont ces pratiques d'intervention que nous présentons dans la partie suivante. ${ }^{11}$

\section{LA MISE EN FORME DES PRATIQUES D'EXCLUSION SCOLAIRE}

La forme scolaire a permis de former une unité par-delà la diversité et la multiplicité des obstacles relevés, et de dégager plusieurs pratiques qui, reproduisant une certaine norme scolaire, empêcheraient les élèves de mobiliser l'aide disponible, c'est-à-dire le capital social accessible. Mises bout à bout, ces pratiques acquièrent une dimension particulière : elles composent une forme d'intervention spécifique qui produirait, de manière non délibérée, des situations d'exclusion scolaire, notamment pour les élèves en situation de vulnérabilité. Il s'agit de pratiques relevant de la promotion d'un parcours d'excellence ou encore de la centration sur la culture scolaire.

\section{La promotion d'un parcours d'excellence scolaire}

Dans le cadre de notre recherche, nous avons pu observer que la promotion d'un parcours d'excellence scolaire pourrait contribuer à exclure des élèves dont les trajectoires ne correspondent pas aux attentes transmises par le prisme de la forme scolaire, en prévenant ainsi la production de capital social. La promotion systématique de cette norme s'incarne de plusieurs manières : à travers l'insistance sur la réussite chiffrée, l'autorité de la note, l'accent mis sur certaines matières scolaires et le renforcement de programmes particuliers.

L'insistance sur la réussite chiffrée. Les données issues des entretiens et les observations des événements concernant les élèves révèlent qu'à l'école Pierre-Mathieu, la réussite est appréhendée principalement à travers le prisme des résultats aux évaluations. Aussi, la rhétorique des évaluations (notamment ministérielles) s'accompagne d'un cérémonial préparé et répété tout au long de l'année par les praticiens - rituel que les élèves eux-mêmes reprennent, voire amplifient, alors que les évaluations plus informelles ne suscitent que très peu d'intérêt pour la plupart d'entre eux. Interrogés au cours des entretiens sur ce que signifie pour eux réussir, plusieurs avancent qu'il s'agit d'" avoir plus de $60 »^{12}$ (Yael), d'" avoir une bonne note " (Edouardo), ou encore que les « bons " élèves sont ceux qui ont "des belles notes " (Roberto). La réussite est ainsi appréhendée principalement à travers le prisme des évaluations chiffrées. D'ailleurs, certains élèves participant à la recherche considèrent que l'obtention de « bonnes " notes est synonyme d'intelligence. 
Cet effet est pour le moins pervers puisqu'à travers le classement qu'elle induit, la note créerait un premier niveau d'exclusion pour ceux qui n'obtiennent pas de " bonnes " notes. Tel est le cas de Yassim, un élève en classe d'adaptation scolaire, qui présente des grandes difficultés scolaires. Il rencontre plusieurs obstacles dans son parcours : il s'est fait renvoyer d'un stage et avoue avoir de la difficulté à gérer sa prise de médicaments (il est diagnostiqué avec un trouble déficitaire de l'attention avec hyperactivité). Toutefois, il revient systématiquement sur ses notes et, à plusieurs reprises dans les données issues des événements significatifs avec les élèves, il avoue qu'il « [n'est] pas intelligent ». C'est d'ailleurs ainsi qu'il justifie ne pas voir l'utilité de mobiliser le capital social accessible (les intervenants, les professeurs ou autres spécialistes auxquels il pourrait avoir recours) : cela ne servirait à rien. Yassim n'est pas le seul à faire ce lien entre les notes et l'intelligence, et d'autres élèves, dans les données issues des événements significatifs, tiennent le même discours dépréciatif. Pour ces élèves, la norme d'excellence transmise par la note opérerait une ségrégation qui aurait des conséquences sur l'estime de soi.

L'autorité de la note. En mettant l'accent sur les résultats scolaires, la note écarterait les autres moyens que les élèves mobilisent et qui peuvent conduire à leur réussite. C'est ce qui ressort des propos de plusieurs élèves en difficulté scolaire au cours des entretiens. Les élèves qui présentent un parcours plus long et complexe sembleraient ainsi se retrouver marginalisés, en périphérie du système. Ils sont pourtant très peu nombreux à contester cet état de fait. C'est toutefois le cas de Roberto et Brandon, deux élèves diagnostiqués avec un trouble d'apprentissage dans deux classes d'adaptation scolaire. Les deux rencontrent des difficultés scolaires, notamment en français (le français est la deuxième langue de Brandon) et sociales (les relations avec leurs enseignants mais aussi avec certains de leurs camarades de classe). Lors d'un même entretien, les deux élèves dénoncent à l'envi l'injustice des notes, et notamment le fait qu'elles ne permettraient pas aux enseignants de tenir compte de leurs efforts (de conduite, notamment), de leur bonne volonté, de leur présence en classe et de l'ensemble des actions qu'ils déploient. Roberto s'explique (avec véhémence) :

l'affaire qu'ils [les enseignants] disent les notes, les notes. Mais arrête de nous parler des notes, tu crois que ça nous encourage?... je me lève le matin. Moi je veux vraiment. Y m'dit tu coules tes notes. Des affaires comme ça. Moi j'me lève le matin, je viens à l'école. Ouais j'ai p't'être pas des bonnes notes, mais au moins j'viens à l'école pour me forcer. Et toi tu m'décourages comme ça?

Les entretiens et les questionnaires révèlent ainsi que Brandon et Roberto ont décidé de rejeter clairement toute aide académique. Brandon avoue ne rien faire en classe tandis que Roberto a décidé de ne plus aller dans certains cours et de ne plus faire ses travaux.

L'accent sur le français et les mathématiques. Certaines matières scolaires, soumises au principe de la sélection, pourraient devenir des marqueurs de la réussite. 
C'est ainsi que le français et les mathématiques tiennent le haut du pavé à l'école Pierre-Mathieu. Il suffit de constater l'espace qui leur est accordé dans les procès-verbaux des rencontres du conseil d'établissement et dans le projet éducatif. Les documents de présentation de l'école et les observations des événements significatifs concernant les acteurs scolaires révèlent également le caractère transversal de ces matières, répété à l'envi (l'importance du français en histoire, des mathématiques en sciences physiques, etc.). La valeur du français et des mathématiques se reflète également dans les propos des élèves au cours des observations et des entretiens (de groupe) et auprès des personnes clés (entretiens spontanés). Même les élèves les plus en difficulté (en français et en mathématiques) semblent d'ailleurs avoir intégré l'aspect primordial de la réussite dans ces deux matières : les observations des événements significatifs les concernant révèlent que les examens ministériels dont ces matières font l'objet provoquent chez eux beaucoup plus de stress que les autres évaluations mises en place dans l'école. Certains élèves en difficulté dans ces matières s'organisent pour réviser ensemble pendant les pauses scolaires et les plus en difficulté demandent aux plus performants sur le plan académique, comme Toufik ou Gaens, de les guider dans leur révision. Toutefois, les questionnaires et les entretiens révèlent qu'ils n'en viennent pas pour autant à mobiliser le capital social mis en place par l'école.

Le renforcement des programmes d'excellence. La documentation liée aux différents programmes offerts par l'école révèle que le français et les mathématiques sont déterminants dans les tests de sélection pour accéder aux différents programmes particuliers de l'école Pierre-Mathieu (multimédia, technologies, communication, etc.); ces mêmes programmes correspondant au parcours typique d'excellence scolaire. Il n'est donc pas étonnant de constater que les cursus qui proposent des mathématiques enrichies y soient tout particulièrement privilégiés. C'est ainsi que l'école favoriserait l'accès des bons élèves (du point de vue scolaire) à ces programmes prestigieux, alors que seulement $40 \%$ se retrouvent dans les programmes dits réguliers. Dans le cadre de cette recherche, ce sont ceux qui réussissent le mieux scolairement qui se destinent à ces programmes. Et certains n'hésitent pas à développer des stratégies pour mobiliser le capital social de l'école à cet effet. Au cours des entretiens et des observations des événements significatifs avec les élèves, Toufik explique sa réaction quand il ne comprend pas une règle en français : il ne va pas seulement voir son enseignant de français actuel mais aussi son enseignante de l'année précédente, avec laquelle il a gardé contact. Selon lui, elle répond davantage à ses attentes.

Les résultats de cette première section s'ancrent dans une perspective plus large. L'insistance sur la réussite chiffrée, les matières d'excellence et les programmes particuliers font partie des mécanismes de sélection des bons élèves (Perrenoud, 1989). Or, cette sélection n'est pas sans conséquence quand on sait que ces choix finissent par créer une sorte d'homogénéisation scolaire qui ne bénéficie pas forcément aux élèves les plus performants mais affaiblit sûrement les résultats 
des élèves les plus faibles (Duru-Bellat, 2009; Merle, 2011). Pour Dubet (1994), ces mécanismes privilégient ainsi les élèves issus des milieux les plus favorisés et dont on prévoit l'excellence des performances parce qu'ils bénéficient d'un soutien familial plus efficace. L'orientation reposerait donc davantage sur la performance que sur des choix véritables, c'est-à-dire les goûts des élèves (Dubet, 1994) ou encore leurs dispositions (Lahire, 2008). Ces mécanismes latents d'orientation permettraient à ceux qui en comprennent les rouages de pouvoir tirer leur épingle du jeu scolaire (Duru-Bellat et Van Zanten, 2012).

\section{La centration sur la culture scolaire}

La centration sur la culture scolaire est un autre mécanisme qui pourrait contribuer à exclure certains élèves. La forme scolaire se montrerait ainsi étanche aux influences et ne se laisserait pas pénétrer par les logiques des acteurs qu'elle accueille, notamment ceux qui ne correspondent pas à la norme transmise. Cette centration sur le mode scolaire, qui prévient la mobilisation du capital social de l'école par les élèves les plus vulnérables, prend plusieurs formes, depuis la priorisation de la mission d'instruction, la restriction de la mission de socialisation, jusqu'à la mise à distance de la culture des élèves.

La priorisation de la mission d'instruction. À l'école Pierre-Mathieu, le projet éducatif, la convention de gestion et de réussite éducative et le plan de réussite révèlent une insistance marquée sur la mission "instruire ", au détriment des autres missions «qualifier " et "socialiser " prescrites par le MEES (2017b). Dans ces documents, l'apprentissage des élèves y est en effet principalement appréhendé à travers le prisme des résultats scolaires. À titre d'exemple, les uniques objectifs du volet " qualifier " du projet éducatif de l'école (sur 5 ans) sont d'" augmenter les taux de réussite des élèves du $1^{1^{\text {er }}}$ et du $2^{\text {e }}$ cycle pour la compétence à résoudre une situation problème » et d' " augmenter les taux de réussite des élèves du $1^{\text {er }}$ et du $2^{\mathrm{e}}$ cycle pour la compétence à déployer un raisonnement mathématique ». Toujours en ce qui a trait à la qualification, les documents de communication et les observations des événements significatifs concernant les intervenants scolaires soulignent que les programmes professionnels techniques courts de l'école (CPF, FMS, FTP) $)^{13}$ ne bénéficient pas du même rayonnement et de la même visibilité que les programmes réguliers et (surtout) les programmes particuliers (multimédia, technologie, etc.). De plus, les observations des événements significatifs avec les élèves montreraient que ces programmes sont nettement moins attractifs et moins privilégiés par les élèves.

Les élèves semblent avoir intégré cette dévaluation du volet " qualifier ». Lors des entretiens et des observations des événements les concernant, ils rejettent l'ensemble des programmes scolaires en lien avec cette qualification. Ils avouent voir le cours de Projet personnel d'orientation, censé justement les aider dans le développement de leur projet professionnel, comme une « joke » ${ }^{14}$ un cours qui ne sert à rien. Ils admettent d'ailleurs " niaiser $»^{15}$ en classe. Les entretiens spontanés auprès des personnes-clés semblent aller dans le même sens alors 
que les deux intervenants communautaires s'interrogent sur le bien-fondé de ces cours. La même logique pourrait également expliquer le désintérêt ou le manque de motivation des élèves à mobiliser certaines ressources sociales en lien avec la qualification, comme le conseiller en orientation ou encore les intervenants censés les aider dans la recherche d'emploi, alors que la plupart d'entre eux affirment pourtant vouloir se trouver un travail.

La restriction de la mission de socialisation. La centration sur la culture scolaire s'incarnerait à travers une certaine déformation de la mission "socialiser ". Dans les documents de présentation de l'école (site et vidéos de présentation), mais également dans le projet éducatif ou encore dans le plan de réussite, cette mission est principalement envisagée à travers le prisme du « climat sécuritaire ». La question du vivre-ensemble serait un exemple de cette distorsion : alors que cette notion est continuellement promue dans les observations des événements significatifs avec les acteurs scolaires (les différentes allocutions de la direction et les discours de présentation de l'école), les documents comme le code de vie, les règles de vie interne, les différents protocoles d'intervention ou encore les documents de présentation de l'école révèlent que ce vivre-ensemble n'a d'autre vertu que d'être un préalable à un environnement sécuritaire. Si les deux termes ("vivre-ensemble " et " sécurité ») ne recouvrent pas les mêmes réalités, ils y sont pourtant à plusieurs reprises associés, comme lorsqu'on évoque le " climat harmonieux et sécuritaire " ou encore le code de vie qui préserve "la paix, le bon ordre et la sécurité ». Selon la même logique, le projet éducatif a pour orientation "le maintien d'un climat harmonieux et sécuritaire " et, à maintes reprises, les documents de présentation soulignent les efforts déployés se concentrant sur l'instauration d'un " environnement scolaire sécuritaire ".

La socialisation devient ainsi une condition du « climat scolaire ", subordonnée à l'apprentissage (à la mission d'instruction). Les réactions des élèves à ce sujet, lors des entretiens et des observations les concernant, pourraient être révélatrices de la restriction de cette mission. Ils se moquent ouvertement des cours qui ont pour objectif d'enseigner la citoyenneté, l'ouverture à l'autre (le cours d'Éthique et culture religieuse notamment), les ateliers de prévention (sur l'homosexualité, le respect, etc.), et tournent en dérision les campagnes contre l'intimidation scolaire. D'autant plus que, d'après les documents officiels et les observations des événements significatifs concernant les intervenants scolaires, la socialisation serait en grande partie confiée aux organismes communautaires, spécialisés en intervention sociale. Leur place semble pourtant nettement moins privilégiée que les intervenants scolaires dont la mission est intégrée à celle de l'école (volet « instruire »). Situés à l'étage des casiers, les organismes en intervention sociale ne jouiraient pas des mêmes avantages en termes de rayonnement et de visibilité : ils ne partageraient pas les mêmes ressources ni les mêmes responsabilités (ils ne disposent que d'un rôle d'observation au conseil d'établissement) et leurs interactions avec les ressources scolaires au sujet des élèves sont quasi-inexistantes (d'ailleurs, ils n'ont pas accès aux dossiers 
des élèves). Toutefois, les questionnaires révèlent que les élèves tendent à les mobiliser davantage que les autres ressources de l'école.

La mise à distance de la culture des élèves. La forme scolaire demeurerait déconnectée du vécu de certains élèves. Une des illustrations repose sur l'absence de place que l'école accorde à leurs intérêts dans les documents de présentation de l'école, dans la documentation en lien avec les programmes ou encore dans les observations des rencontres du conseil d'établissement. À titre d'exemples, les observations des événements significatifs avec les acteurs scolaires révèlent que le sport, la musique ou les réseaux sociaux sont des activités très peu valorisées dans le cadre scolaire et déléguées en grande partie aux intervenants communautaires. De même, les requêtes des élèves (par exemple, agrandir la salle de jeux, le café jeunesse, etc.) relevées au cours des observations des événements les concernant et lors des entretiens sembleraient trouver très peu d'écho auprès des instances scolaires quand elles ne sont pas conformes à la forme scolaire. ${ }^{16}$ Autre manifestation de la distance avec la forme scolaire : lors des entretiens, plusieurs élèves qui présentent des difficultés scolaires insistent sur le manque de sens et d'utilité des matières scolaires (les mathématiques et le français) dans la "vraie vie ». Dario s'explique :

un exemple que j'ai trouvé c'est on te montre comment trouver un inconnu dans une équation à x... à plusieurs variables... tu vas pas faire ça dans la vraie vie, si tu peux trouver facilement, tu vas pas te compliquer la vie.... Alors parfois t'as l'impression d'apprendre quelque chose inutilement, pis ça te démoralise, tu perds la motivation pis le truc.

À cela s'ajoute que les entretiens et les observations des événements significatifs avec les élèves révèlent que les attentes de l'école sur le plan comportemental ne seraient pas toujours évidentes, notamment pour les élèves les plus vulnérables. L'ajustement face à ces règles implicites semble se retrouver chez des élèves qui, sous prétexte de bien se conduire (ayant parfaitement conscience de l'importance accordée à la conduite des élèves), se permettent d'enfreindre des règles académiques de l'école sans s'attirer de punition. Ainsi, Edouardo a des "bonnes notes " et sait s'attirer la sympathie des enseignants; il peut traîner dans les couloirs, s'endormir en classe et arriver en retard à son cours lorsqu'il sort de sa pratique de football sans risquer de se voir infliger une punition. Ce n'est pas le cas de St-Martin, un élève en CPF, dont la conduite est considérée du point de vue de l'institution comme plus difficile à gérer et dont les retards à répétition lui attirent immanquablement des heures de récupération.

Ceux parmi les élèves qui réussissent sur le plan scolaire, comme Toufik ou Gaens, ${ }^{17}$ semblent les plus stratégiques tandis que les élèves qui présentent un parcours plus long et hésitant se retrouveraient plus souvent marginalisés. Lors des entretiens, c'est ce que met en relief un dialogue au cours duquel Toufik recommande à Roberto, dubitatif, d'être gentil pour avoir des bonnes notes. Selon lui « au Canada, ok ici, l'école elle est vraiment facile, même si 
t'es poche, mais le prof voit que t'essaies de faire des efforts, il va te faire passer ». Dans cet échange, on est en présence de deux attitudes très distinctes : celle de Toufik, stratégique, qui pense voir clair dans le jeu des ajustements entre les conduites scolaires et sociales (ce qui semble lui réussir d'après les observations des événements significatifs concernant les élèves), et celle de Roberto, sceptique, qui semble ne pas comprendre cette manouvre entre la règle sociale et la règle scolaire.

Plusieurs théoriciens (Maulini et Perrenoud, 2005; Vincent, 2008) s'interrogent sur les évolutions de la forme scolaire et se demandent si celle-ci est en train de s'ouvrir, c'est-à-dire se laisser pénétrer par les logiques des acteurs qu'elle accueille. Les résultats de cette recherche semblent pousser à répondre par la négative et aller dans le même sens que Maulini et Perrenoud (2005), qui dénoncent un "déni " des multiples connaissances et compétences dont sont porteurs les élèves quand celles-ci n'ont pas de pertinence au regard de l'excellence scolaire. Au niveau linguistique (Bakhtine, 1984) et cognitif (Bauthier et Goigoux, 2004), certains auteurs utilisent le terme "secondarisation " pour expliquer ce processus de mise à distance. Cette secondarisation peut conduire l'école à catégoriser certains élèves simplement parce que leur culture diffère de la culture institutionnelle, une ségrégation d'autant plus importante que l'école Pierre-Mathieu semblerait évacuer les dimensions sociales (avec la socialisation) qui entrent en jeu dans les difficultés scolaires, contribuant ainsi à individualiser la réussite et l'échec scolaires.

\section{CONCLUSION}

Dans le cadre de cet article, nous avons décrit plusieurs pratiques scolaires qui participeraient de l'exclusion des élèves les plus vulnérables, en appréhendant l'exclusion comme absence de production de capital social. Ainsi, la promotion d'un parcours d'excellence et la centration sur la culture scolaire constituent autant de facettes d'une forme scolaire spécifique, normative et prescriptive, qui fonctionnerait en vase clos, en ce sens qu'elle empêcherait les élèves de mobiliser l'aide disponible, c'est-à-dire le capital social organisationnel. Cette forme scolaire rendrait ainsi légitimes les projets des élèves en accord avec ses modalités mêmes d'application, ${ }^{18}$ alors qu'elle contribuerait à exclure les élèves qui ne lui correspondent pas. Mises bout à bout, les différentes pratiques répertoriées dans cet article constituent autant de situations qui, en se superposant, finiraient par sédimenter une forme de ségrégation scolaire des élèves en difficulté et ainsi «fabriquer " l'échec scolaire (Perrenoud, 1994). Il importe dans ce sens de prendre en compte ces différents éléments. Ils constituent les principaux obstacles qui se dressent pour atteindre une véritable inclusion qui répond aux réalités de tous les élèves et notamment des plus vulnérables.

Néanmoins, trois nuances sont ici nécessaires. Il convient tout d'abord de mentionner que ces processus ne sont pas toujours rationnels et participent 
ainsi des « conséquences non intentionnelles de l'action " (Giddens, 1987, p. 41). Cette exclusion n'est pas volontaire et les différentes situations qui y conduisent partent souvent d'un principe de bienveillance, voire d'une intention d'inclusion. C'est la mise en place cumulative des actions qui finit par nuire à ceux qu'elle vise, plutôt que de les aider, contribuant ainsi à ce que Deniger, Lemire et Germain (2016) nomment la "bienveillance morbide " (p. 114). Par ailleurs, il faut rappeler que cette forme scolaire ne repose pas sur une simple imposition; ses mécanismes relèvent plutôt d'une construction sociale, interactive et dynamique, créant parfois une sorte d'aliénation avec une reprise, voire parfois une amplification, du discours par certains acteurs. Selon ce principe, les élèves qui rencontrent des difficultés peuvent ainsi se retrouver aliénés au discours de la forme scolaire, coincés entre un sentiment d'incapacité qui va jouer sur leur estime de soi et une révolte inféconde qui accentue leur exclusion. Ce point renvoie d'ailleurs à une limite de cette recherche : même si certains intervenants scolaires ont participé à la recherche par le biais des entretiens spontanés, le fait de pouvoir entrer dans les salles de classe aurait permis de mieux comprendre les négociations que les enseignants et orthopédagogues font de cette forme scolaire.

Une part de solution est enfin apportée par certains programmes de formation qui découlent des politiques éducatives, comme la formation interculturelle (Mc Andrew, Borri-Anadon, Larochelle-Audet et Potvin, 2013; Steinbach, 2012) ou encore la pédagogie de l'inclusion (Bergeron et St-Vincent, 2011). Ces programmes procurent des indications précises pour orienter l'école québécoise sur le chemin de l'inclusion. En s'écartant de la norme du groupe majoritaire, les pratiques que ces programmes préconisent favoriseraient ainsi la médiation entre la forme scolaire et la réalité des élèves, facilitant ainsi la production du capital social dans l'école, notamment pour les élèves les plus vulnérables. Selon cette perspective, l'inclusion ne se limiterait pas forcément à un partage de normes et valeurs communes mais relèverait davantage d'une meilleure compréhension et d'une prise en compte accrue des besoins multiples de tous les élèves. Il est toutefois nécessaire de placer ces programmes dans un contexte qui va au-delà des pratiques qu'ils promeuvent afin d'appréhender l'inclusion et l'exclusion scolaires de manière systémique, en tenant compte de l'influence potentielle des facteurs politiques et sociaux externes. La compétition privé-public, l'importance grandissante accordée au palmarès des écoles (Desjardins, Lessard et Blais, 2009), les mécanismes de gestion de la Gestion axée sur les résultats (GAR; Maroy, 2017) ou encore les compressions budgétaires vécues par les écoles au cours des dernières années (Nadeau, 2016) ont en effet une incidence significative et peuvent donc in fine affecter l'objectif d'inclusion scolaire. 


\section{NOTES}

1. La vulnérabilité est ici appréhendée comme la résultante de situations problématiques. Dans le cadre scolaire, ces situations peuvent être pensées comme des obstacles à la scolarité (et donc à la réussite) d'élèves qui présentent des conditions initiales défavorables (comme des élèves présentant un handicap [HDAA], des élèves issus de l'immigration, des élèves en situation de grande précarité, etc.).

2. Dans cette perspective, l'exclusion est définie comme un « processus de rupture du lien social, désaffiliation " et repose sur le fait que " les personnes ne peuvent pas pleinement participer ou contribuer à la société à cause du déni de droits politiques, civils, sociaux, économiques et culturels " (UNESCO, 2017, paragr. 1).

3. L'ensemble des éléments concernant l'école (nom, données propres à l'école, etc.) est rendu anonyme afin qu'elle ne puisse être identifiée.

4. L'école se situe dans le décile le plus élevé (10/10) en ce qui concerne l'indice de faible revenu (SFR) et l'indice de milieu socio-économique (IMSE).

5. Par exemple, les rencontres du conseil d'établissement, les soirées de rencontres parents-enseignants, les rencontres d'intervenants, les journées de formation, les ateliers et interventions, etc.

6. Parmi le corpus de documents officiels citons : les procès-verbaux et autres documents informatifs délivrés au cours des différentes réunions des Conseils éducatifs, les plans de réussite, le projet éducatif, les conventions de gestion et de réussite éducative, le code de vie, les messages officiels des directions, les documents adressés aux parents, les documents de la CSDM approuvés et repris par l'école, les documents scolaires concernant l'ensemble des élèves de l'école (liste des élèves, codage des difficultés, profils pédagogiques, etc.).

7. Il s'agit d'une organisatrice communautaire (1 entretien), d'un surveillant (1 entretien), d'une enseignante d'accueil (1 entretien), d'un intervenant communautaire (2 entretiens), d'un intervenant communautaire (1 entretien), d'un ancien élève de l'école (1 entretien), d'un éducateur spécialisé (1 entretien) et d'une bénévole (1 entretien).

8. Par exemple, l'aide aux devoirs, les ateliers offerts aux élèves, les rencontres d'élèves autour de leurs travaux scolaires, les diverses sollicitations des élèves envers certains intervenants, etc.

9. Les questionnaires se sont inspirés des questionnaires d'analyse des réseaux sociaux (Burt, 2000). Ils avaient pour objectif d'identifier les ressources que les élèves mobilisaient quand ils faisaient face à des difficultés de différents ordres : scolaire, affectif, social et matériel. Ces difficultés avaient au préalable été listées au cours des observations.

10. Nous reprenons la définition des représentations de Jodelet (2003), qui appréhende les représentations comme « une forme de connaissance socialement élaborée et partagée, ayant une visée pratique et concourant à la construction d'une réalité commune à un ensemble social» (p. 53).

11. Les pratiques dites de gestion de la difficulté (le modèle médical de l'échec scolaire, la relégation des élèves vers des programmes particuliers, l'externalisation de la prise en charge de la difficulté, l'organisation en silo de la remédiation) font l'objet d'un autre article. Cette division a entraîné un certain nombre de modifications purement formelles et n'a aucune incidence sur le fond de la recherche.

12. L'utilisation des guillemets renvoie aux citations des élèves lors des entretiens et des documents de l'école.

13. Ce sont des classes de pré-spécialisation qui proposent des formations courtes aux élèves en difficulté. Elles portent plusieurs noms : Cheminement particulier de formation (CPF), Formation préparatoire au travail (FPT), Formation à un métier semi-spécialisé (FMS).

14. C'est-à-dire comme une plaisanterie.

15. S'amuser.

16. Les observations des évènements significatifs concernant les élèves soulignent que les demandes de type plus scolaire (par exemple ouvrir un local pour l'étude, disposer de plus de temps de travail, etc.) semblent recevoir un accueil plus favorable. 
17. D'ailleurs, ces élèves sont souvent montrés en exemple par l'école (voir Gaens, qui se trouve même sur le site de l'école).

18. Comme mentionné dans les résultats, à travers l'évaluation, l'accès aux programmes particuliers ou à certains privilèges dont bénéficient les élèves les plus stratégiques.

\section{RÉFÉRENCES}

Archambault, J., Garon, R. et Harnois, L. (2011). Diriger une école en milieu défavorisé. Des caractéristiques des écoles performantes, provenant de la documentation scientifique. Montréal, QC : Université de Montréal et Une école montréalaise pour tous.

Aucoin, A. et Vienneau, R. (2015). Inclusion scolaire et dénormalisation. Dans N. Rousseau (dir.), La pédagogie de l'inclusion scolaire : pistes d'action pour apprendre tous ensemble (p. 63-86). Québec, QC : Presses de l'Université du Québec.

Bakhtine, M. (1984). Esthétique de la création verbale. Paris, France : Gallimard.

Bautier, E. et Goigoux, R. (2004). Difficultés d'apprentissage, processus de secondarisation et pratiques enseignantes : une hypothèse relationnelle. Revue Française de Pédagogie, 148, 89-99.

Becker, H. S. (1963). Outsiders. Studies in the sociology of deviance. Oxford, Royaume-Uni : Free Press of Glencoe.

Bergeron, G. et St-Vincent, L.-A. (2011). L'intégration scolaire au Québec : regard exploratoire sur les défis de la formation à l'enseignement au primaire et préscolaire. Éducation et francophonie, 39(2), 272-295.

Bevort, A. et Lallement, M. (2006). Le capital social. Performance, équité et réciprocité. Paris, France : La Découverte.

Borri-Anadon, C. (2015). Entrele diree le faire: pratiquesévaluatives des orthophonistes à l'égard des élèves issus de minorités culturelles [Fiches de lecture du CEETUM]. Repéré à http://www.francaislangueseconde. fr/wp-content/uploads/2017/06/BORRI-ANADONorthophoniste-pratique-minorite-culturelle.pdf

Burt, R. S. (2000). The network structure of social capital. Dans R. I. Sutton et B. M. Staw (dir.), Research in organizational behavior (p. 345-423). Greenwich, CT : Jai Press.

Conseil supérieur de l'éducation (CSE). (2017). Desécoles inspirantes qui s'adaptent à la diversité des élèves. Repéré à http://cse.gouv.qc.ca/fichiers/documents/publications/EtudesRecherches/50-0505.pdf

Deniger, M-A. (2012). Les politiques québécoises d'intervention en milieux scolaires défavorisés : regard historique et bilan critique. Revue française de pédagogie, 178, 67-84.

Deniger, M-A, Lemire, V. et Germain, L. (2016). La part de l'école dans l'inclusion scolaire et dans la construction des inégalités scolaires [Rapport soumis à la Commission scolaire de Montréal]. Montréal, QC : CSDM.

Desjardins, P-D., Lessard, C. et Blais, J-G. (2011). Les effets prédits et observés du Bulletin des écoles secondaires du Québec. Dans Y. Dutercq (dir.), Où va l'éducation entre public et privé? (p. 105-123). Paris, France : De Boeck.

Dika, S. L. et Singh, K. (2002). Applications of social capital in educational literature: A critical synthesis. Review of Educational Research, 72(1), 31-60.

Dubet, F. (1994). Sociologie de l'expérience. Paris, France : Seuil.

Duru-Bellat, M. (2002). Les inégalités sociales à l'école : genèse et mythes. Paris, France : Presses universitaires de France.

Duru-Bellat, M. (2009). Inégalités sociales à l'école: du constat aux politiques. Dans B. Toutlemonde (dir.), Le système éducatif en France (p. 269-279). Paris, France : La Découverte.

Duru-Bellat, M. et Van Zanten, A. (2012). Sociologie de l'école. Paris, France : Armand-Colin.

Institut Fraser (2016). Le Bulletin des écoles secondaires du Québec. Performance des écoles. Repéré à https://www.fraserinstitute.org/fr/\%C3\%A9tudes/bulletin-des-ecoles-secondaires-du-quebec-2016 


\section{De la fabrication de l'exclusion scolaire en milieu défavorisé et multiethnique}

Giddens, A. (1987). La constitution de la société. Paris, France : PUF.

Institut national d'excellence en santé et en services sociaux (INESSS). (2018). Évaluation de l'efficacité des interventions psychosociales pour les enfants, adolescents et jeunes adultes ayant un trouble de déficit de l'attention avec ou sans hyperactivité ou des difficultés apparentées. Montréal, QC : Auteur.

Jodelet, D. (2003). Les représentations sociales. Paris, France : Presses universitaires de France.

Kamanzi, C., Zhang, X. Y., Deblois, L. et Deniger, M. A. (2007). L'influence du capital social sur la formation du capital humain chez les élèves résilients de milieux socioéconomiques défavorisés. Revue des sciences de l'éducation, 33(1), 127-145.

Lahire, B. (2008). La forme scolaire dans tous ses états. Schweizerische Zeitschrift für Bildungswissenschaften, 30(2), 229-258.

Lareau, A. et Dorvat, E. (1999). Moments of social inclusion and exclusion race, class, and cultural capital in family-school relationships. Sociology of Education, 72(1), 37-53.

Leana, C. R. et Pil, F. K. (2014, 14 octobre). A new focus on social capital in school reform efforts. [Billet de blogue]. Repéréà http://www.shankerinstitute.org/blog/new-focus-social-capital-school-reform-efforts

Lemire, V. (2010). Obstacles à la persévérance scolaire d'adultes ayant des problèmes d'apprentissage lors de leur passage à l'éducation des adultes (Mémoire de maîtrise inédit). Université de Montréal, Montréal, QC.

Lin, N. (1995). Les ressources sociales : une théorie du capital social. Revue française de sociologie, 36(4), 685-704.

Magnan, M.-O., Pilote, A., Vidal, M. et Collins, T. (2016). Le processus de construction des étiquettes dans les interactions scolaires. Dans M. Potvin, M.-O. Magnan et J. Larochelle-Audet (dir.), La diversité ethnoculturelle, religieuse et linguistique en éducation. Théorie et pratique (p. 231-237). Anjou, QC : Fides Éducation.

Mc Andrew, M., Borri-Anadon, C., Larochelle-Audet, J. et Potvin, M. (2013). La formation initiale du personnel scolaire sur la diversité ethnoculturelle, religieuse et linguistique dans les universités québécoises : un premier bilan. Dans M. Mc Andrew, M. Potvin et C. Borri-Anadon (dir.), Le développement d'institutions inclusives en contexte de diversité. Recherche, formation, partenariat. Québec, QC : Presses de l'Université du Québec.

Maroy, C. (2017). La nouvelle gestion publique de l'école au Québec : vers une gestion de la pédagogie. Sociologie du travail, 59(4). Repéré à https://journals.openedition.org/sdt/1353

Maulini, O. et Perrenoud, P. (2005). La forme scolaire de l'éducation de base : tensions internes et évolutions. Dans O. Maulini et C. Montandon (dir.), Les formes de l'éducation : variété et variations (p. 147-168). Bruxelles, Belgique : De Boeck.

Merle, P. (2011). Concurrence et spécialisation des établissements scolaires. Une modélisation de la transformation du recrutement social des secteurs d'enseignement public et privé. Revue française de sociologie, 52, 133-169.

Merriam, S. (1998). Qualitative research and case study applications in education. San Francisco, CA : Jossey-Bass.

Ministère de l'Éducation et de l'Enseignement supérieur (MEES). (2017a). Indices de défavorisation. Repéré à http://www.education.gouv.qc.ca/references/tx-solrtyperecherchepublicationtx-solrpublicationnouveaute/resultats-de-la-recherche/detail/article/indices-de-defavorisation/

Ministère de l'Éducation et de l'Enseignement supérieur (MEES). (2017b). Orientations pédagogiques. Repéré à http://www.education.gouv.qc.ca/eleves/lecture-a-lecole/bibliotheques-scolaires/ pourquoi-une-bibliotheque/orientations-pedagogiques/

Ministère de l'Éducation, du Loisir et du Sport (MELS). (2007). L'organisation des services éducatifs aux élèves à risque et aux élèves handicapés ou en difficulté d'adaptation ou d'apprentissage (EHDAA). Québec, QC : Gouvernement du Québec.

Ministère de l'Éducation du Québec (MEQ). (1998). Politique d'intégration scolaire et d'éducation interculturelle. Montréal, QC : Gouvernement du Québec. 
MEQ. (1999). Une école adaptée à tous ses élèves : prendre le virage du succès - Politique de l'adaptation scolaire. Québec, QC : Gouvernement du Québec.

Nadeau, J. (2016, 16 mars). Compressions et déficits. Le Devoir. Repéré à https://www.ledevoir.com/ societe/education/465585/education-depenses-dans-le-reseau-de-l-education

Paillé, P. et Mucchielli, A. (2012). L'analyse qualitative en sciences humaines et sociales. Paris, France : Armand Colin.

Parsons, T. (1959). Élément pour une sociologie de l'action. Paris, France : Plon.

Perrenoud, P. (1989). La triple fabrication de l'échec scolaire. Paris, France: Presses universitaires de France.

Perrenoud, P. (1990). Culture scolaire, culture élitaire. Coordination, 37, 21-33.

Perrenoud, P. (1994). Métier d'élève et sens du travail scolaire. Paris, France : Éditions Sociales Françaises.

Pil, F. K. et Leana, C. (2009). Applying organizational research to public school reform: The effects of teacher human and social capital on student performance. Academy of Management Journal, 52(6), 1101-1124.

Portes, A., (1998). Social capital: Its origins and applications in modern sociology. Annual Review of Sociology, 24(1), 1-24.

Prud'homme, L. (2015). Se former à un enseignement pour tous les élèves. Dans N. Rousseau (dir.), La pédagogie de l'inclusion scolaire : pistes d'action pour apprendre tous ensemble (p. 345-372). Québec, QC : Presses de l'Université du Québec.

Quintero, E. (2014, 17 juillet). Do students learn more when their teachers work together? [Billet de blogue]. Repéré à http://www.shankerinstitute.org/blog/do-students-learn-more-when-their-teachers-work-together

Spillane, J. P. (2015, 15 avril). Broadening the educational capability conversation: Leveraging the social dimension [Billet de blogue]. Repéré à http://www.shankerinstitute.org/blog/broadening-educational-capability-conversation-leveraging-social-dimension

Stanton-Salazar, R. D. (2011). A social capital framework for the study of institutional agents and their role in the empowerment of low-status students and youth. Youth et Society, 43(3), 1066-1109.

Steinbach, M. (2012). Élargir les perspectives interculturelles des futurs enseignants. McGill Journal of Education, 47(2), 147-265.

Tondreau, J. et Robert. M. (2011). L'école québécoise. Débat, enjeux et pratiques sociales. Anjou, QC: CEC.

Tumin, M. M. (1967). Social stratification: The forms and functions of inequality. Gembloux, Belgique : Duculot.

UNESCO. (2017). Exclusion. Apprendre à vivre ensemble. Repéré à http://www.unesco.org/new/ $\mathrm{fr} /$ social-and-human-sciences/themes/international-migration/glossary/exclusion/

Vidal, M. (2017). Le capital social comme outil d'intervention : accès et mobilisation dans une école secondaire en milieu défavorisé et multiethnique (Thèse de doctorat inédite). Université de Montréal, QC.

Vincent, G. (1980). L'école primaire française. Étude sociologique. Lyon, France : Presses Universitaires de Lyon.

Vincent, G. (2008). La socialisation démocratique contre la forme scolaire. Éducation et francophonie, 36(2), 47-62.

White, M. et Glick, J. (2000). Generation status, social capital, and the routes out of high school. Sociological Forum, 15(4), 671-691.

Yan, W. (1999). Successful African American students: The role of parental involvement. The Journal of Negro Education, 68(1), 5-22.

Zhang, X., DeBlois, L., Deniger, M. A. et Kamanzi, C. (2008). A theory of success for disadvantaged children: Re-conceptualization of social capital in the light of resilience. Alberta Journal of Educational Research, 54(1), 97-111. 
MARJORIE VIDAL est stagiaire postdoctorale au département d'éducation et formation spécialisées de l'Université du Québec à Montréal (UQAM). Elle détient un doctorat en administration et fondements de l'éducation (Université de Montréal) et a réalisé un postdoctorat (Actions concertées - FRQSC) avec le Centre d'études et de recherches sur les transitions et l'apprentissage (CÉRTA), dans le département d'orientation de l'Université de Sherbrooke. L'inclusion scolaire et sociale est au cœur des thématiques qui l'intéressent. Son cheminement l'a conduite à s'intéresser à l'étude de cas ethnographique pour son apport à l'approche d'intervention sociale. vidal.marjorie@uqam.ca

MARJORIE VIDAL is a postdoctoral fellow in the department of education and special training at Université du Québec à Montréal (UQAM). She holds a doctorate in administration and foundations of education (Université de Montréal) and received a postdoctoral fellowship (Actions concertées - FRQSC) to work with the Centre d'études et de recherches sur les transitions et l'apprentissage (CÉRTA), in the department of vocational education and training at Université de Sherbrooke. Inclusive education and social inclusion are at the center of her main fields of interest. Her career path led her to focus on ethnographic case study for its contribution to social intervention. vidal.marjorie@uqam.ca 